\title{
The Largest Component of Near-Critical Random Intersection Graph with Tunable Clustering
}

\author{
Shiying Huang $\mathbb{D}^{1}$ and Bin Wang $\mathbb{D}^{2}$ \\ ${ }^{1}$ School of Mathematics and Computational Science, Xiangtan University, Xiangtan, Hunan 411105, China \\ ${ }^{2}$ College of Science, Guilin University of Technology, Guilin, Guangxi 541004, China \\ Correspondence should be addressed to Bin Wang; binwangglut@163.com
}

Received 26 August 2021; Accepted 16 October 2021; Published 9 November 2021

Academic Editor: Barbara Martinucci

Copyright (c) 2021 Shiying Huang and Bin Wang. This is an open access article distributed under the Creative Commons Attribution License, which permits unrestricted use, distribution, and reproduction in any medium, provided the original work is properly cited.

In this paper, we study the largest component of the near-critical random intersection graph $G(n, m, p)$ with $n$ nodes and $m$ elements, where $m=\Theta(n)$ which leads to the fact that the clustering is tunable. We prove that with high probability the size of the largest component in the weakly supercritical random intersection graph with tunable clustering on $n$ vertices is of order $n \epsilon(n)$, and it is of order $\epsilon^{-2}(n) \log \left(n \epsilon^{3}(n)\right)$ in the weakly subcritical one, where $\epsilon(n) \longrightarrow 0$ and $n^{1 / 3} \epsilon(n) \longrightarrow \infty$ as $n \longrightarrow \infty$.

\section{Introduction and Main Result}

Singer-Cohen [1] and Karoński et al. [2] invented the binomial random intersection graph model $G(n, m, p)$. It can be constructed by a random bipartite graph $\mathscr{B}(n, m, p)$, which is a graph with vertex set $V=\left\{v_{1}, \ldots, v_{n}\right\}$ and element set $M=\left\{u_{1}, \ldots, u_{m}\right\}$. Any vertex $v_{i}$ and any element $u_{j}$ are independently connected with probability $p$ in $\mathscr{B}(n, m, p)$, $i=1, \ldots, n, j=1, \ldots, m$. Then $G(n, m, p)$ is a random graph with the vertex set $V$, and the vertices $v_{i}$ and $v_{k}$ are connected if both $v_{i}$ and $v_{k}$ are adjacent to an element in $\mathscr{B}(n, m, p)$. Since then, random intersection graphs have received considerable attention in the past two decades. They have been widely used for classification analysis $[3,4]$, clustering [5,6], wireless sensor networks [7], social networks [8], and so on; refer to the survey [9] and references therein for more information on their application.

One of the important issues in random graphs is to determine the size of the largest component. Denote the size of the largest component of $G(n, m, p)$ by $\left|\mathscr{C}_{1}(G(n, m, p))\right|\left(\left|\mathscr{C}_{1}\right|\right.$ for simplicity). Behrisch [10] found that when $m=\left\lfloor n^{\alpha}\right\rfloor$ (when $m>1$ and it is not an integer, $G(n, m, p)$ means $G(n,\lfloor m\rfloor, p)$ with $\lfloor m\rfloor$ being the largest integer less than $m$ ) and $p=c / \sqrt{n m}$, there is a phase transition of $\left|\mathscr{C}_{1}\right|$ at the point $c=1$. When $\alpha>1$, if $p>1 / \sqrt{n m}$, then with high probability (for a given graph property $\mathscr{A}$, the authors say that graph $G_{n}$ possesses $\mathscr{A}$ with high probability if $\lim _{n \longrightarrow \infty} \mathbf{P}\left(G_{n}\right.$ possesses $\left.\mathscr{A}\right)=1$ ) (w.h.p. for brevity) $\left|\mathscr{C}_{1}\right|$ is linear in $n$; if $p<1 / \sqrt{n m}$, then w.h.p. its order is $\log n$. Meanwhile, when $0<\alpha<1$, it jumps from $n^{(1-\alpha) / 2} \log n$ to $n^{(1+\alpha) / 2}$ as $c$ increases. Lagerås and Lindholm [11] extended this result to the case when $m=\beta n$ and $p=\gamma / n$, whose clustering is tunable [6]. For the first rigorous mathematical analysis of clustering in random intersection graphs, see [12]. Recently, Wang et al. [13,14] studied the component evolution in $G(n, m, p)$ when $p=1 \pm \epsilon(n) / \sqrt{n m}$, where $m=$ $n^{\alpha}(\alpha>1)$ and $\epsilon(n) \longrightarrow 0$ as $n \longrightarrow \infty$. They obtained that the order of $\left|\mathscr{C}_{1}\right|$ and the width of the scaling window around the critical probability depend on whether $\alpha \geq(5 / 3)$. If $\alpha \geq(5 / 3)$, they are $n^{2 / 3}$ and $n^{-(1 / 3)}$, respectively, which are independent of $\alpha$, and the evolution is similar to that in the critical Erdös-Rényi random graph; meanwhile if $1<\alpha<5 / 3$, they are $n^{(3-\alpha) / 2}$ and $n^{-(\alpha-1 / 2)}$, respectively. Federico [15] obtained that when $\alpha>1$ $(0<\alpha<1$, respectively), the sizes of the largest components of critical $G(n, m, p)$, rescaled by $n^{(-2 / 3)}\left(n^{-(3-\alpha / 6)}\right.$, respectively), converge to nondegenerated random variables. It is natural to ask what is the order of $\left|\mathscr{C}_{1}\right|$ near the critical window in $G\left(n,\lfloor\beta n\rfloor, \gamma n^{-1}\right)$, that is, when $\beta \gamma^{2}=1 \pm \epsilon(n)$, where $\epsilon(n)$ $\longrightarrow 0$ as $n \longrightarrow \infty$. Now our main result can be stated as follows. 
Theorem 1. Let $\beta$ be a positive constant which is independent of $n$ with $\gamma:=\gamma(n)$ being a positive function on $n$. Denote $\epsilon(n)$ by a positive function on $n$ such that $\epsilon(n) \longrightarrow 0$ and $n^{1 / 3} \epsilon(n) \longrightarrow \infty$ as $n \longrightarrow \infty$. In $G(n, m, p)$ with $m=\lfloor\beta n\rfloor$ and $p=\gamma / n$, the following statements hold:

(1) (Weakly supercritical regime). If $\beta \gamma^{2}=1+\epsilon(n)$, then there are two positive constants $C_{1}$ and $C_{2}$ which are independent of $n$ such that w.h.p.

$$
C_{1} n \epsilon(n) \leq\left|\mathscr{C}_{1}\right| \leq C_{2} n \epsilon(n) \text {. }
$$

(2) (Weakly subcritical regime). If $\beta \gamma^{2}=1-\epsilon(n)$, then there are two positive constants $C_{3}$ and $C_{4}$ which are independent of $n$ such that w.h.p.

$$
C_{3} \epsilon^{-2}(n) \log \left\{n \epsilon^{3}(n)\right\} \leq\left|\mathscr{C}_{1}\right| \leq C_{4} \epsilon^{-2}(n) \log \left\{n \epsilon^{3}(n)\right\} .
$$

Remark 1. Let $\epsilon(n)>0$ and $\lim \epsilon(n)=0$. If $n \epsilon^{3}(n) \longrightarrow \infty$, then in the Erdös-Rényi random graph $G(n,(1-\epsilon(n) / n))$ w.h.p. the size of the largest component is $(2+o(1)) \epsilon^{-2}(n) \log \left(n \epsilon^{3}(n)\right)$, and in $G(n,(1+\epsilon(n) / n))$, w.h.p. it is $(2+o(1)) n \epsilon(n)$; while if $n \epsilon^{3}(n)=O(1)$, then in $G(n,(1 \pm \epsilon(n) / n))$ w.h.p. it is of order $n^{2 / 3}$. So it is worth showing that the upper bound and lower bound can be approximately equivalent near the critical window in $G(n,\lfloor\beta n\rfloor, \gamma / n)$. It is interesting to determine the size of largest component in $G(n,\lfloor\beta n\rfloor, \gamma / n)$ when $\beta \gamma^{2}=1 \pm \epsilon(n)$ and $\epsilon(n)=O\left(n^{-(1 / 3)}\right)$. It may be of size approximately $n^{(2 / 3)}$. The method used in this article failed to prove these two problems.

Remark 2. For the upper bound of the size of largest component in random graphs, one of standard methods is to use a branching process to dominate the corresponding exploration process from above. Usually the main problem is how to obtain the lower bound. To bound $\left|\mathscr{C}_{1}\right|$ from below in $G(n,\lfloor\beta n\rfloor, p)$, it does not work by the comparison of $G(n, m, p)$ and ErdösRényi random graph $G(n, \widehat{p})$ for some suitable $\widehat{p}$ through the result in [16], as it does not cover the case when $m$ and $n$ are of the same order. Hence, first we converted the problem of bounding $\left|\mathscr{C}_{1}\right|$ from below into the problem of giving a lower bound for the size of the largest component in random bipartite graph $\mathscr{B}(n, n, p)$. Then we analyzed the Depth First Search (DFS) algorithm and an exploration process on $\mathscr{B}(n, n, p)$ to achieve our purpose. Note that Johannson [17] only proved that the order of the largest component in the critical $\mathscr{B}(n, n, p)$ is of order $o(n)$. We think this is our main contribution.

Remark 3. When we decided to submit it to a journal for possible publication, Ambroggio [18] proposed an elementary approach to study the upper bound of the component size in some critical random graph. We applied her/ his argument in Section 3.1 and found that it is shorter than our proof. While the method in [18] does not work for the case $m=n^{\alpha}(\alpha>1)$, the branching process argument works. So we present it here instead of the proof using Ambroggio's argument.
Our notations are standard. $\mathbf{P}(\cdot), \mathbf{E}(\cdot)$, and $\operatorname{Var}(\cdot)$ denote the probability, expected value, and variance, respectively. For any two positive functions $f(n)$ and $g(n)$, write $f(n)=$ $O(g(n))$ or $g(n)=\Omega(f(n))$ if $f(n) \leq C g(n)$ for some positive constant $C$ when $n$ is large enough; $f(n)=\Theta(g(n))$ if $f(n)=O(g(n))$ and $g(n)=O(f(n)) ; \quad$ and $f(n)=o(g(n))$ or $g(n) \gg f(n)$ if $\lim _{n \longrightarrow \infty} f(n) / g(n)=0$. All logarithms have the natural base, and all inequalities or asymptotic statements are to be understood in terms of $n$ being large enough yet fixed. For clarity of presentation, write $\epsilon(n)=\epsilon$, and floor and ceiling signs are omitted whenever they are not essential in the rest of the paper.

The remainder of this paper is organized as follows. In Section 2, for the lower bound of $\left|\mathscr{C}_{1}\right|$, we first show that it is only needed to give a lower bound for the size of the largest component in $\mathscr{B}(n, n, p)$. Then, we obtain the lower bound by analyzing the DFS algorithm on $\mathscr{B}(n, n, p)$ for the weakly supercritical regime in Section 2.1 and by analyzing the standard exploration process on $\mathscr{B}(n, n, p)$ for the subcritical one in Section 2.2. We get the upper bound for the weakly supercritical regime in Section 3.1 and that for the weakly subcritical one in Section 3.2 through the branching process method and the coupling technique, respectively.

\section{The Proof of the Lower Bound}

In this section, the following standard exploration process on the random bipartite graph $\mathscr{B}(n, m, p)$ will be used.

For exploration process $\left\{Y_{t}\right\}_{t \geq 0}$, let $\mathscr{C}(v)$ be the component containing the vertex $v \in V$ in $\mathscr{B}(n, m, p)$. In this procedure, the vertices and the elements will be in an active, inactive, or neutral state. In the beginning, it is assumed that all vertices and elements are neutral. We choose a vertex, say $v_{0}$, from $V$ uniformly and make it active. At each time $t \geq 1$, we choose a vertex $v_{t}$ or element $u_{t}$ uniformly from the active vertices and elements. Suppose that we choose a vertex $v_{t}$, check the pairs $v_{t} u^{\prime}$ where $u^{\prime}$ runs over all the neutral elements. If $v_{t} u^{\prime} \in \mathscr{B}(n, m, p)$, then make $u^{\prime}$ active; otherwise, keep it neutral. After checking all the neutral elements, let $v_{t}$ be inactive. Meanwhile if we choose an element $u_{t}$, check the pairs $u_{t} v^{\prime}$ where $v^{\prime}$ runs over all the neutral vertices. If $u_{t} v^{\prime} \in \mathscr{B}(n, m, p)$, then make $v^{\prime}$ active; otherwise, keep it neutral. When there is no active vertex or element, component $\mathscr{C}\left(v_{0}\right)$ is explored which is composed of vertices and elements in $\mathscr{B}(n, m, p)$. Then, we uniformly choose a neutral vertex (or an element) from the rest of the neutral ones and proceed on.

For simplicity, we do not distinguish the vertices and elements and call them vertices in this section. It is natural as we only consider the size of the largest component in $\mathscr{B}(n, m, p)$. So let $X_{t}$ be the number of vertices which become active due to the exploration of vertex $v_{t}\left(v_{t} \in V\right.$ or $\left.v_{t} \in M\right)$; and let $Y_{t}$ be the total number of active vertices at step $t \in\{0,1, \ldots, n\}$, where $Y_{0}=1$. It is easy to see that, for any $t \geq 1$,

$$
Y_{t}= \begin{cases}Y_{t-1}+X_{t}-1, & \text { if } Y_{t-1}>0 \\ X_{t}, & \text { if } Y_{t-1}=0\end{cases}
$$

Now we can obtain the following Lemma 1 and Lemma 2 by applying the above process on $\mathscr{B}(n, \beta n, p)$. 
Lemma 1. For any $p \in(0,1)$, let $\left|\mathscr{C}_{1}\left(G\left(n, \beta_{i} n, p\right)\right)\right|$ be the size of the largest component in $G\left(n, \beta_{i} n, p\right)$, where $\beta_{i}$ is a positive constant which is independent of $n, i=1,2$. If $0<\beta_{1} \leq \beta_{2}$, then

$$
\left|\mathscr{C}_{1}\left(G\left(n, \beta_{1} n, p\right)\right)\right|<\left|\mathscr{C}_{1}\left(G\left(n, \beta_{2} n, p\right)\right)\right| .
$$

Here see the footnote for the relation $\prec$ (the authors say random variable $X$ is stochastically smaller than random variable $Y$ and denote it by $X \prec Y$, if and only if there exists a coupling $(\widehat{X}, \widehat{Y})$ of $X$ and $Y$ such that $\mathbf{P}(\widehat{X} \leq \widehat{Y})=1)$.

Proof. Let $V:=\left\{v_{1}, \ldots, v_{n}\right\}$ which is the vertex set of $G\left(n, \beta_{1} n, p\right)$ and $G\left(n, \beta_{2} n, p\right)$. Suppose that the element sets of $G\left(n, \beta_{1} n, p\right)$ and $G\left(n, \beta_{2} n, p\right)$ are $M_{1}$ and $M_{2}$, respectively, where $M_{1}=\left\{u_{1}, \ldots, u_{\beta_{1} n}\right\}$ and $M_{2}=\left\{u_{1}, \ldots, u_{\beta_{1} n}\right.$, $\left.u_{\beta_{1} n+1}, \ldots, u_{\beta_{2} n}\right\} \supseteq M_{1}$. Given the random bipartite graph $\mathscr{B}\left(n, \beta_{2} n, p\right)$ with sets $V$ and $M_{2}$, the graph $\mathscr{B}\left(n, \beta_{1} n, p\right)$ is defined to be the subgraph of $\mathscr{B}\left(n, \beta_{2} n, p\right)$ induced by $V$ and $M_{1}$. By this construction, the probability of $v_{\ell}$ and $v_{k}$ which are adjacent in $G\left(n, \beta_{2} n, p\right)$ is larger than that in $G\left(n, \beta_{1} n, p\right)$. Then the conclusion holds obviously.

Lemma 2. Let $\left|\mathscr{C}_{1}(\mathscr{B}(n, \beta n, p))\right|$ be the size of the largest component in $\mathscr{B}(n, \beta n, p)$ for any $p \in(0,1)$, where $\beta$ is a positive constant which is independent of $n$. Denote the number of its vertices by $\left|\mathscr{C}_{1}^{\beta}(V)\right|$ which is just $\left|\mathscr{C}_{1}(G(n, \beta n, p))\right|$ and the number of its elements by $\left|\mathscr{C}_{1}^{\beta}(M)\right|$. If $\mid \mathscr{C}_{1}(\mathscr{B}$ $(n, \beta n, p)) \mid:=f(n) \longrightarrow+\infty$ as $n \longrightarrow \infty$, then w.h.p.

$$
\left|\mathscr{C}_{1}^{\beta}(V)\right|=\left|\mathscr{C}_{1}(G(n, \beta n, p))\right|=\Theta(f(n)) \text { and }\left|\mathscr{C}_{1}^{\beta}(M)\right|=\Theta(f(n)) \text {. }
$$

Proof. In the process of exploring $\mathscr{B}(n, \beta n, p)$, suppose that the explored element set and the active element set at time $t$ are $M_{D}^{(t)}$ and $M_{A}^{(t)}$, respectively, and the explored vertex set and active vertex set are $V_{D}^{(t)}$ and $V_{A}^{(t)}$, respectively. If we choose an active vertex $v_{t}$ from $V$ at time $t$, then the number of elements which would be explored through $v_{t}$ (denote it by $\left.\left|X^{(t)}\right|\right)$ is distributed as the binomial distribution $\operatorname{Bin}\left(\beta n-\left|M_{D}^{(t)}\right|-\left|M_{A}^{(t)}\right|, p\right)$. Therefore, $\left|X^{(t)}\right|<\Upsilon_{t}$ for any $t \leq \min \{n, T\}$, where $T=\min \left\{t: Y_{t}=0\right\}$ and $\Upsilon_{t}$ is distributed as $\operatorname{Bin}(\beta n, p)$.

Now, suppose that we get the largest component $\mathscr{C}_{1}(\mathscr{B}(n, \beta n, p))$ in which there are $\left|\mathscr{C}_{1}^{\beta}(V)\right|=g(n)$ vertices from $V$ and $\left|\mathscr{C}_{1}^{\beta}(M)\right|$ elements from $M$ through the exploration process. Conditioned on $\left|\mathscr{C}_{1}^{\beta}(V)\right|=g(n)$, we can determine what the order of $\left|\mathscr{C}_{1}^{\beta}(M)\right|$ should be. In fact, by the above explanation, the number of elements which would be explored through any vertex $v \in V$ is dominated by $\operatorname{Bin}(\beta n, p)$ at any step $t$; and remember that we made the assumption that there are $g(n)$ vertices from $V$. Hence, for the total number of elements $\left|\mathscr{C}_{1}^{\beta}(M)\right|$ we explored, we have

$$
\left|\mathscr{C}_{1}^{\beta}(M)\right|=\sum_{t=1}^{g(n)} X^{(t)} \prec \Upsilon_{1}+\Upsilon_{2}+\cdots+\Upsilon_{g(n)} \prec \Upsilon .
$$

Here $\Upsilon$ is distributed as $\operatorname{Bin}(g(n) \beta n, p)$. It is easy to check

$$
\begin{aligned}
\mathbf{E}(\Upsilon) & =\beta \gamma g(n) \longrightarrow \infty ; \\
\operatorname{Var}(\Upsilon) & =o\left((\mathbf{E}(\Upsilon))^{2}\right) .
\end{aligned}
$$

Chebyshev's inequality (refer to [[19], Corollary 4.3.3]) implies $\Upsilon=(1+o(1)) \beta \gamma g(n)$. Hence w.h.p. $\left|\mathscr{C}_{1}^{\beta}(M)\right| \leq(1+o(1)) \beta \gamma g(n)$. Notice $\beta \gamma=\sqrt{\beta}+o(1)$ under the assumption that $\beta \gamma^{2}=1+o(1)$. We have

$$
\begin{aligned}
g(n) & \leq f(n)=\left|\mathscr{C}_{1}(\mathscr{B}(n, \beta n, p))\right|=\left|\mathscr{C}_{1}^{\beta}(V)\right|+\left|\mathscr{C}_{1}^{\beta}(M)\right| \\
& \leq g(n)+(1+o(1)) \beta \gamma g(n)=(1+\sqrt{\beta}+o(1)) g(n) .
\end{aligned}
$$

The proof of $\left|\mathscr{C}_{1}^{\beta}(M)\right|=\Theta(f(n))$ can be given similarly.

Recall that the component in $\mathscr{B}(n, m, p)$ consists of some vertices from $V$ and some elements from $M$. According to Lemma 1 , when $\beta \geq 1$, we only need to bound $\left|\mathscr{C}_{1}(G(n, n, p))\right|$ from below; and when $\beta \leq 1$, we choose an element from $M$ and run the exploration process on $\mathscr{B}(n, \beta n, p)$. Then it boils down to the case where $\beta \geq 1$ by Lemma 2 . Therefore, we only need to give a lower bound on $\left|\mathscr{C}_{1}(\mathscr{B}(n, n, p))\right|$ in the rest of this section. Note that in Theorem 1 when $m=n, p=\sqrt{1 \pm \epsilon} / n=1 \pm \epsilon / 2+o(\epsilon) / n$. We write $p=1 \pm \epsilon / n$ for simplicity as it does not affect the result when $\epsilon \longrightarrow 0$ and $\epsilon n^{1 / 3} \longrightarrow \infty$.

2.1. Lower Bound in the Weakly Supercritical Regime. Breadth First Search (BFS) and Depth First Search (DFS) are two well-known algorithms in the graph exploration. They are widely used to study the structure of (random) graphs. The DFS has been utilized to investigate random graphs by some scholars such as Ben-Eliezer et al. [20], Krivelevich and Sudakov [21], Joseph [22], and Cooley et al. [23]. In this section, we applied the DFS to the bipartite graph $B \in \mathscr{B}(n, n, p)$ to obtain the lower bound according to the ideas in $[21,23]$ which are used to study the largest component in the Erdös-Rényi random graph and the random hypergraph, respectively. Before starting to prove the lower bound, we need a lemma about a sequence of i.i.d. Bernoulli random variables with parameter $p$.

Lemma 3. Let $\left\{\kappa_{i}\right\}_{i=1}^{n^{2}}$ be the i.i.d. Bernoulli random variables with parameter $p=1+\epsilon / n$, where $\epsilon$ is a positive function on $n$ such that $\epsilon \longrightarrow 0$ and $\epsilon n^{1 / 3} \longrightarrow \infty$ as $n \longrightarrow \infty$. Then, for any $t \in\left[a_{1} \in n^{2}, a_{2} \in n^{2}\right]$, where $a_{i}$ s are positive constants independently of $n, i=1,2$, w.h.p. we have

$$
\left|\sum_{i=1}^{t} \kappa_{i}-p t\right| \leq \frac{\epsilon^{2} n}{4}
$$

The following auxiliary lemma is needed to prove Lemma 3. 
Lemma 4 (see Lemma 7 in [24] and Lemma 9 in [23]). Let $\left(Z_{i}\right)_{i=1}^{\ell}$ be a martingale with $Z_{0}=0$. If there are two positive constants $b_{1}$ and $b_{2}$ with $b_{1}<b_{2} / 10$ such that $-b_{1} \leq Z_{i}-Z_{i-1} \leq b_{2}$ for any $i \in[1, \ell]$, then, for any $0<C<b_{1} \ell$,

$$
\mathbf{P}\left(\left|Z_{\ell}\right| \geq C\right) \leq 2 \exp \left\{-\frac{C^{2}}{3 b_{1} b_{2} \ell}\right\}
$$

Proof of Lemma 3. For any $t \in\left[a_{1} \epsilon n^{2}, a_{2} \epsilon n^{2}\right]$, define $\Lambda_{0}=0$, and

$$
\Lambda_{t+1}= \begin{cases}\Lambda_{t}+\kappa_{t+1}-p, & \text { if }\left|\Lambda_{t}\right| \leq \frac{\epsilon^{2} n}{4} \\ \Lambda_{t}, & \text { otherwise. }\end{cases}
$$

It is easy to verify that it indeed is a martingale with the stopping time $\min \left\{t:\left|\Lambda_{t}\right|>\varepsilon^{2} n / 4\right\}$; and $-p \leq \Lambda_{t+1}-\Lambda_{t} \leq$ $1-p<1$. By Lemma 4 , we have

$$
\begin{aligned}
\mathbf{P}\left(\left|\sum_{i=1}^{t} \kappa_{i}-p t\right| \geq \frac{\epsilon^{2} n}{4}\right) & \leq 2 \exp \left\{-\frac{\left(\epsilon^{2} n\right)^{2}}{48 p a_{2} \epsilon n^{2}}\right\} \\
& =2 \exp \left\{-\frac{n \epsilon^{3}}{48 a_{2}(1+\epsilon)}\right\}=o(1) .
\end{aligned}
$$

Now we prove the lower bound of $\left|\mathscr{C}_{1}(\mathscr{B}(n, n, p))\right|$.

Proof. We choose a graph $B$ from $\mathscr{B}(n, n, p)$ uniformly. Write $B=(V, M, E)$, where $E$ is the edge set. Let $\sigma_{V}$ be a linear ordering of $V$ and let $\sigma_{M}$ be a linear ordering of $M$. In the exploration process, let $S$ be the set of vertices that have been explored; let $T$ be the set of unvisited vertices, and $U=V \cup M \backslash(S \cup T)$ in which the vertices of $U$ are put in a stack. We run the following DFS algorithm on $B$ to obtain components (recall that both the vertices and the elements are called vertices in this section). See Algorithm 1.

Consider a sequence $\left\{\kappa_{i}\right\}_{i=1}^{n^{2}}$ of the i.i.d. Bernoulli random variables with parameter $p$ which is the sequence of edges in $B \in \mathscr{B}(n, n, p)$. That is, $\kappa_{i}$ is the result of the $i$-th queried edge. If the queried edge is in $B$, then $\kappa_{i}=1$; otherwise $\kappa_{i}=0$. We have the following claim.

Claim 1. Between the query $4 \epsilon n^{2} / 5$ and the query $\epsilon n^{2}$ w.h.p. the stack $U$ is not empty.

Proof of Claim. First, we can assume that $|S|<n$. In fact, if $|S| \geq n$, let us consider the time that $|S|=n$. By Lemma 3, $|U|<2 a_{2} \epsilon n$, which means $|T|=2 n-|S|-|U|>n-2 a_{2} \epsilon n$. Since $B$ is a bipartite graph, when we run the DFS on $B$, both $S$ and $T$ have half vertices in $V$ and $M$. Hence, between $S$ and $T$, at time $t$ the DFS has made queries at least
$2 \times\left(\frac{|S|}{2}-1\right) \times\left(\frac{|T|}{2}-1\right) \geq 2\left(\frac{n}{2}-1\right)\left(\frac{n-2 a_{2} \epsilon n}{2}-1\right)>\frac{n^{2}}{4} \gg \epsilon n^{2} \geq t$

It is a contradiction. Secondly, by Lemma 3, we have that, at any time $t \in\left[4 \epsilon n^{2} / 5, \epsilon n^{2}\right],|S \cup U| \geq p t-\epsilon^{2} n / 4$. Suppose that stack $U$ is empty which means there are only explored vertices and neutral vertex after some $t$ queries for $t \in\left[4 \epsilon n^{2} / 5, \epsilon n^{2}\right]$; then $|S| \geq p t-\epsilon^{2} n / 4$. Therefore, if stack $U$ is empty, we can assume $|S| \epsilon\left(p t-\left(\epsilon^{2} n / 4\right), n\right)$. Meanwhile, at time $t$, the number of edges that have been queried is w.h.p. at least $|S| / 2(n-|S| / 2) \times 2$, which comes from the fact that when we get set $S$ by running DFS on the bipartite graph $B$ we have about $|S| / 2$ vertices from $V$ and $|S| / 2$ elements from $M$, and every vertex (resp. element) has $n-|S| / 2$ elements (resp. vertices) to be paired. Note that $x(n-x / 2)$ is an increasing function when $x \in\left(p t-\left(\epsilon^{2} n / 4\right), n\right)$. This means that

$$
\begin{aligned}
t & \geq \frac{|S|}{2}\left(n-\frac{|S|}{2}\right) \times 2 \geq\left(p t-\frac{\epsilon^{2} n}{4}\right)\left(n-\frac{p t}{2}+\frac{\epsilon^{2} n}{8}\right) \\
& =(1+\epsilon) t-\frac{(1+\epsilon)^{2} t^{2}}{2 n^{2}}+\frac{(1+\epsilon) \epsilon^{2} t}{4}-\frac{\epsilon^{2} n^{2}}{4}-\frac{\epsilon^{4} n^{2}}{32} \\
& \geq t+\frac{4 \epsilon^{2} n^{2}}{5}-\frac{(1+\epsilon)^{2} \epsilon^{2} n^{2}}{2}+\frac{(1+\epsilon) \epsilon^{3} n^{2}}{5}-\frac{\epsilon^{2} n^{2}}{4}-\frac{\epsilon^{4} n^{2}}{32} \\
& =t+\epsilon^{2} n^{2}\left(\frac{11}{20}-\frac{(1+\epsilon)^{2}}{2}+o(1)\right)>t .
\end{aligned}
$$

It is a contradiction!

Now, we proceed on the proof of the lower bound. The Claim and Lemma 3 imply that w.h.p. the number of $\kappa_{i}=1$ between $4 \epsilon n^{2} / 5$ and $\epsilon n^{2}$ is at least

$$
p € n^{2}-\frac{\epsilon^{2} n}{4}-\left(p \frac{4 \epsilon n^{2}}{5}+\frac{\epsilon^{2} n}{4}\right)=\left(\frac{1}{5}+o(1)\right) € n \text {. }
$$

That is, all the number of vertices added to $U$ in the interval $\left[4 \epsilon n^{2} / 5, \epsilon n^{2}\right]$ is at least $(1 / 5+o(1)) \epsilon n$. Obviously, these vertices are in the same component. Therefore, $\left|\mathscr{C}_{1}(\mathscr{B}(n, n, p))\right|=\Omega(\epsilon n)$.

2.2. Lower Bound in the Weakly Subcritical Regime. Before beginning to prove the lower bound, we need a lemma borrowed from [25].

Lemma 5 (see Lemma 3.1 in [25]). Let $\left\{\chi_{i}\right\}$ be a sequence of random variables with the binomial distribution $\operatorname{Bin}(k, p)$, where $p=1-\epsilon / k$. Define a process $\left\{W_{t}\right\}_{t \geq 0}$ as $W_{t}=W_{t-1}+$ $\chi_{t}-1$ with $W_{0}=1$. Set

$$
\tau:=\min \left\{t: W_{t}=0\right\} .
$$

Then there exist two positive constants $c_{1}, c_{2}$ such that, for all $T_{0} \geq \epsilon^{-2}$, 
Input: $B=(V, M, E), \sigma_{V}$-a linear ordering of $V, \sigma_{M^{-}}$a linear ordering of $M$.

Output: $\mathscr{C}$-set of components of $B$.

$\mathscr{C}:=\varnothing$; The stack $U:=\varnothing$;

\section{repeat}

Let $v_{0}$ be the smallest neutral vertex in $V$ according to $\sigma_{V}$;

Make $v_{0}$ as active, and add $v_{0}$ to $U$

Whenever $U \neq \varnothing$ do

Let $x$ be the top vertices of $U$. Scanning T.

If $x \in V$ and there exists the smallest element $u$ according to $\sigma_{M}$

such that $x u \in E$ then delete $x$ from $T$ and put it into $U$.

Otherwise, then take $x$ out of $U$ and put it into $S$.

If $x \in M$ and there exists the smallest vertex $v$ according to $\sigma_{V}$

such that $x v \in E$ then delete $x$ from $T$ and put it into $U$.

Otherwise, then take $x$ out of $U$ and put it into $S$.

Let $C$ be the set of vertices explored in the while-loop above;

$\mathscr{C}:=\mathscr{C} \cup C$

Until all vertices are explored.

Algorithm 1: DFS on bipartite graph $B \in \mathscr{B}(n, n, p)$ starting from a vertex in $V$.

$$
\mathbf{P}\left(\tau \geq T_{0}\right) \geq c_{1}\left(\epsilon^{-2} T_{0}^{-(3 / 2)} e^{-\left(\epsilon^{2}+c_{2} \epsilon^{3}\right) T_{0} / 2}\right)
$$

Proof of the Lower Bound in the Weakly Subcritical Case. Our proof follows the line in [25]. We analyzed the exploration process defined at the beginning of this section. Recall that $X_{t}$ is the number of vertices which become active due to the exploration of active vertex $v_{t}$, and $Y_{t}$ is the total number of active vertices at step $t \in\{0,1, \ldots, n\}$. For the exploration process $\left\{Y_{t}\right\}_{t \geq 0}$, let $t_{1}<t_{2}<\cdots$ be the times at which $Y_{t}=0$. Then $\left(t_{1}, t_{2}-t_{1}, \ldots, t_{i}-t_{i-1}, \ldots\right)$ are the sizes of the components in $\mathscr{B}(n, n, p)$. It is easy to see that the total neutral vertices at time $t$ are

$$
N_{t}=n-t-Y_{t} \text {. }
$$

For small enough $\delta \in(0,1)$, define a stopping time $\Lambda$ with respect to $N_{t}$ as

$$
\Lambda:=\min \left\{t: N_{t} \leq n-\delta \epsilon n\right\} .
$$

Set $L_{t}:=Y_{t}-Z_{t}$, where $Z_{t}:=\sum_{i=1}^{t-1} 1_{\left\{Y_{t}=0\right\}}$. We can see that $Z_{t}$ counts the number of components completely explored by the process before time $t$. For any integer $j$, let $W_{t}^{(j)}$ be a process with increments distributed as $\operatorname{Bin}(n-\delta \epsilon n, p)$, and its starting point $W_{0}^{(j)}=L_{t_{j}}=-(j-1)$. Let

$$
\tau_{j}:=\min \left\{t: W_{t}^{(j)}=-j\right\} .
$$

Choose a constant $\delta_{1}<2(1-\delta) /(\delta+2)^{2}$, and take

$$
T_{0}=\delta_{1} \epsilon^{-2} \log \left(n \epsilon^{3}\right), N=\epsilon^{-1}\left(n \epsilon^{3}\right)^{1-\delta} .
$$

First, we shall show that w.h.p. the two following events $\mathscr{A}_{1}$ and $\mathscr{A}_{2}$ hold:

$$
\begin{aligned}
& \mathscr{A}_{1}:=\left\{t_{N} \leq \Lambda\right\} ; \\
& \mathscr{A}_{2}:=\left\{\text { there exists } k_{1}<N \text { such that } \tau_{k_{1}}>T_{0}\right\} .
\end{aligned}
$$

Note that $\mathscr{A}_{1} \cap \mathscr{A}_{2} \subset\left\{\left|\mathscr{C}_{1}(\mathscr{B}(n, n, p))\right|>T_{0}\right\}$, where $\left|\mathscr{C}_{1}(\mathscr{B}(n, n, p))\right|$ is the size of largest component in $\mathscr{B}(n, n, p)$. In fact, let $\left\{\alpha_{i}\right\}_{i=1}^{t}$ be a sequence of the i.i.d. random variables distributed as $\operatorname{Bin}(n, p)$. It is easy to get that $X_{i} \prec \alpha_{i}$ for all $i$. Hence by (3) and (19) we have $N_{t} \geq n-1-t-\sum_{i=1}^{t} \alpha_{i}$. Meanwhile $\sum_{i=1}^{t} \alpha_{i} \sim \operatorname{Bin}(n t, p)$ and $\mathbf{E}\left(\sum_{i=1}^{t} \alpha_{i}\right)=(1+\epsilon) t<2 t$. By the Chernoff bounds (refer to Theorem A.1.4 in [19]), there exists a constant $c>0$ such that

$$
\mathbf{P}\left(\sum_{i=1}^{t} \alpha_{i}>3 t\right) \leq e^{-c t}
$$

which means $\mathbf{P}\left(N_{t} \geq n-4 t\right)$ with probability at least $1-e^{-c t}$ leading to $\mathbf{P}\left(N_{t} \leq n-5 t\right) \leq e^{-c t}$. Therefore,

$$
\mathbf{P}\left(\Lambda \leq \frac{\delta € n}{5}\right) \leq e^{-c \epsilon n} \text {. }
$$

Define

$$
W_{t}^{\prime}=W_{t-1}^{\prime}+\alpha_{i}-1=\sum_{i=1}^{t}\left(\alpha_{i}-1\right)
$$

with $W_{0}^{\prime}=1$; and let $\tau^{\prime}:=\min \left\{t: W_{t}^{\prime}=0\right\}$. Then, by Wald's equation (refer to Theorem 4.1.5 in [26]), we have $\mathbf{E} \tau \prime \prime=\epsilon^{-1}$. Recall that when $t<\tau:=\min \left\{t: Y_{t}=0\right\}, Y_{t}=\sum_{i=1}^{t}\left(X_{i}-1\right)$ by [3]. Hence, $\mathbf{E}\left(t_{i+1}-t_{i}\right) \leq \epsilon^{-1}$ which means

$$
\mathbf{E}\left(t_{N}\right) \leq N \epsilon^{-1} \leq \epsilon^{-2}\left(n \epsilon^{3}\right)^{1-\delta} .
$$

Therefore, by Markov's inequality,

$$
\mathbf{P}\left(t_{N}>\frac{\delta \epsilon n}{5}\right) \leq \frac{5 \mathrm{E}\left(t_{N}\right)}{\delta \epsilon n} \leq \frac{5 \epsilon^{-2}\left(n \epsilon^{3}\right)^{1-\delta}}{\delta \epsilon n}=\frac{5\left(n \epsilon^{3}\right)^{-\delta}}{\delta} \longrightarrow 0,
$$

which implies w.h.p. $\mathscr{A}_{1}$ holds. As for $\mathscr{A}_{2}$, when $k=n-\delta \epsilon n$, $p=1-\epsilon(k) / k=1-\epsilon(n-\delta \epsilon n) / n(1-\delta \epsilon) \geq 1-(\delta+2) \epsilon / n$; Lemma 5 indicates that, for any $j$, 


$$
\mathbf{P}\left(\tau_{j}>T_{0}\right) \geq c_{1}^{\prime} \epsilon\left(\log n \epsilon^{3}\right)^{-(3 / 2)}\left(n \epsilon^{3}\right)^{-\delta_{1}(\delta+2)^{2} / 2},
$$

where $c_{1}^{\prime}=c_{1}^{\prime}\left(c_{1}, \delta\right)$. Set $p_{T_{0}}=\mathbf{P}\left(\tau_{j}>T_{0}\right)$. Let $\zeta:=\sum_{i=1}^{N} \zeta_{i}$, where $\zeta_{i}=1$ if $\tau_{i}>T_{0}$; otherwise, $\zeta_{i}=0$. Then $\mathbf{E}(\zeta)=N p_{T_{0}}$ and $\operatorname{Var}(\zeta)=N p_{T_{0}}\left(1-p_{T_{0}}\right)$. Hence, by the Second Moment Method (refer to [[19], Theorem 4.3.1]) and Lemma 5,

$$
\mathbf{P}(\zeta=0) \leq \frac{\operatorname{Var}(\zeta)}{(\mathbf{E} \zeta)^{2}} \leq \frac{1}{N p_{T_{0}}} \leq \frac{1}{c_{1}^{\prime}\left(\log n \epsilon^{3}\right)^{-(3 / 2)}\left(n \epsilon^{3}\right)^{1-\delta-\delta_{1}(\delta+2)^{2} / 2}} \longrightarrow 0
$$

$$
\begin{aligned}
& \text { As } \mathscr{A}_{1} \cap \mathscr{A}_{2} \subset\left\{\left|\mathscr{C}_{1}(\mathscr{B}(n, n, p))\right|>T_{0}\right\}, \\
& \left\{\left|\mathscr{C}_{1}(\mathscr{B}(n, n, p))\right| \leq T_{0}\right\}\left\{\subseteq \overline{\mathscr{A}_{1}} \cup \overline{\mathscr{A}_{2}}=\left\{t_{N}>\Lambda\right\} \cup\{\zeta=0\},\right.
\end{aligned}
$$

which indicates

$$
\mathbf{P}\left\{\left|\mathscr{C}_{1}(\mathscr{B}(n, n, p))\right| \leq T_{0}\right\} \leq \mathbf{P}\left\{t_{N}>\Lambda\right\}+\mathbf{P}\{\zeta=0\}=o(1) .
$$

\section{The Upper Bound}

In this section, we distinguish the vertices and the elements of $\mathscr{B}(n, m, p)$, not like what we do in Section 2. We will make use of the following exploration process on $\mathscr{B}(n, m, p)$. This process is a little different from the exploration process $\left\{Y_{t}\right\}_{t \geq 0}$.

For exploration process $\left\{Y_{t}^{*}\right\}_{t \geq 0}([14])$, let $\mathscr{C}(v)$ be the component containing vertex $v$ in $G(n, m, p)$. In this process, we only care about the vertices from $V$ in $\mathscr{B}(n, m, p)$. So the vertices will be active, inactive, or neutral, but all the elements in $M$ will always be neutral. At the beginning, assuming all the vertices are neutral, we uniformly choose a vertex $v_{0} \in V$ and make it active. At each time $t \geq 1$, we uniformly choose a vertex $v_{t}$ from the active vertices and check all pairs $v_{t} u$ where $u$ runs over all the neutral elements in $M$. If $v_{t} u$ is an edge in $\mathscr{B}(n, m, p)$, then check $u v$ where $v$ runs over all the neutral vertices. If $v_{t} u$ and $u v$ for some element $u$, both are present in $\mathscr{B}(n, m, p)$ and then make $v$ active; otherwise, keep it neutral. After checking all the neutral vertices, let $v_{t}$ be inactive. When there is no active vertex, component $\mathscr{C}\left(v_{0}\right)$ is explored. Then, we choose a neutral vertex uniformly from the rest of the neutral ones in $V$ and proceed on.

Let $X_{t}^{*}$ be the number of vertices which become active due to the exploration of vertex $v_{t}$. Note that $X_{t}^{*}$ is the number of vertices at distance 2 from $v_{t}$ in $\mathscr{B}(n, m, p)$. Denote by $Y_{t}^{*}$ the total number of active vertices at step $t$, where $Y_{0}^{*}=1$. It is easy to see that, for any $t \geq 1$,

$$
Y_{t}^{*}= \begin{cases}Y_{t-1}^{*}+X_{t}^{*}-1, & \text { if } Y_{t-1}^{*}>0 \\ X_{t}^{*}, & \text { if } Y_{t-1}^{*}=0\end{cases}
$$

Define

$T^{*}=\min \left\{t: Y_{t}^{*}=0\right\}=\min \left\{t: X_{1}^{*}+X_{2}^{*}+\cdots+X_{t}^{*}=t-1\right\}$.
At time $T^{*}$, the set of explored vertices is precisely $\mathscr{C}\left(v_{0}\right)$, which means $\left|\mathscr{C}\left(v_{0}\right)\right|=T^{*}$.

The following auxiliary contents would be used in the proof:

(i) Let $\widetilde{X}^{*}$ be a random variable with compound binomial distribution $\mathbf{C B}(n, m, p)$ which has probability generating function

$$
f_{\tilde{X}^{*}}(x)=\mathbf{E}\left[x^{\tilde{X}^{*}}\right]=\left(1-p+p(1-p+p x)^{n}\right)^{m} .
$$

Lemma 6 (see Lemma 2 in [14]). Let $\widetilde{X}_{i}^{*}$ be a random variable distributed as $\mathbf{C B}\left(n_{i}, m, p\right), i=1,2$. If $n_{1} \leq n_{2}$, then $\widetilde{X}_{1}^{*} \prec \widetilde{X}_{2}^{*}$.

(ii) For small enough $x>0$,

$$
\begin{gathered}
1-n x+\frac{n^{2} x^{2}}{2}-n^{3} x^{3}<(1-x)^{n}<1-n x+\frac{n^{2} x^{2}}{2} \\
e^{x}<1+x+x^{2} \\
(1+x)^{k}<1+k x+\frac{2 k^{2} x^{2}}{3} .
\end{gathered}
$$

3.1. Upper Bound in the Weakly Supercritical Regime. The branching process method is widely used to show the upper bound of the size of largest component in random graphs; see $[27,28]$ and references therein. In this section, we applied the branching process argument [29] to obtain the upper bound in the weakly supercritical regime. Kang et al. [30] and Wang and Zou [14] used this technique to get the upper bound of the size of largest component in the corresponding random graphs.

Outline of the proof is as follows: given a vertex $v$ of $G(n, m, p)$, let $\mathscr{C}_{v}$ denote the component of $G(n, m, p)$ containing $v$; and let $\mathscr{T}_{v}$ be the random spanning tree rooted at $v$ which is obtained by exploring new neighbors in $\mathscr{C}_{v}$ via the exploration process $\left\{Y_{t}^{*}\right\}_{t \geq 0}$. Let $\mathscr{B} P$ be the branching process whose offspring distribution is $\mathbf{C B}(n, m, p)$. This process can be interpreted as a potentially infinite random rooted tree. Suppose that there are $\ell$ active vertices and $k$ explored vertices at the time step $t$ in the exploration process on $\mathscr{B}(n, m, p)$. We can see that the number of newly 
explored vertices $X_{t}^{*}$ through the current vertex, say $v_{i}$, has the distribution $\mathbf{C B}(n-k-\ell, m, p)$. Therefore, by Lemma 6, $X_{t}^{*} \prec \xi_{t}$, where $\xi_{t}$ is distributed as $\mathbf{C B}(n, m, p)$, which means the random rooted trees $\mathscr{T}_{v}$ and $\mathscr{B} P$ can be coupled so that $\mathscr{T}_{v}\left\{\subseteq \mathscr{B} P\right.$. Hence, $\left|\mathscr{C}_{v}\right|=\left|\mathscr{T}_{v}\right| \leq|\mathscr{B} P|$, where $|\mathscr{B} P|$ is the size of total offspring in $\mathscr{B} P$. Therefore, we only need to give the upper bound of $|\mathscr{B} P|$.

Before we state some properties about the process $\mathscr{B} P$, recall Theorem 2 .

Theorem 2 (see Theorem 3.7 in [28]). Let $\mathscr{B P}$ be the Galton-Watson branching process with supercritical offspring distribution $\left\{p_{k}\right\}_{k \geq 0}$. Here "supercritical" means $\sum_{k \geq 0} k p_{k}>1$. Suppose that $p_{0} \in(0,1)$, and let $\eta^{*}$ be the extinction probability of $\mathscr{B} P$. Define

$$
q_{k}:=p_{k}\left(\eta^{*}\right)^{k-1}, \quad k \geq 0 .
$$

Then

$$
\sum_{k \geq 0} q_{k}=1 \text { and } \sum_{k \geq 0} k q_{k}=\sum_{k \geq 0} k p_{k}\left(\eta^{*}\right)^{k-1}<1 .
$$

Hence $\left\{q_{k}\right\}_{k \geq 0}$ is an offspring distribution of a subcritical branching process which is often called the dual process of $\mathscr{B} P$. Furthermore, if $\mathscr{T}_{p}$ denotes the branching tree for $\left\{p_{k}\right\}_{k \geq 0}$ and $\mathscr{T}_{q}$ is the corresponding object for $\left\{q_{k}\right\}_{k \geq 0}$, then, for any finite tree $\mathscr{T}$,

$$
\mathbf{P}\left(\mathscr{T}_{p}=\mathscr{T} \mid \mathscr{B} P \text { is extincted }\right)=\mathbf{P}\left(\mathscr{T}_{q}=\mathscr{T}\right) .
$$

Now we can get Lemma 7.

Lemma 7. Let $\mathscr{B} P$ be a branching process with offspring distribution $\mathbf{C B}(n, m, p)$, let $|\mathscr{B} P|$ be its total size, and let $\rho^{*}$ be its surviving probability, where $m, n, p$ satisfy the conditions in Theorem 1. Denote its dual process by $\mathscr{B} P^{*}$ and the total size of $\mathscr{B} P^{*}$ by $\left|\mathscr{B} P^{*}\right|$. Then

(1) $\rho^{*}=(2+o(1)) \epsilon / 1+\gamma$

(2) $\mathbf{E}\left(\left|\mathscr{B} P^{*}\right|\right) \leq 2 / \epsilon$

(3) For any integer $L=L(n), \quad \mathbf{P}(|\mathscr{B} P| \geq L) \leq(1+$ $o(1))[2 \epsilon /(1+\gamma)+2 /(\epsilon L]$

Proof

(1) Theorem 1.6.1 in [31] states that, for the Galton-Watson branching process with offspring distribution $F(x)$, the surviving probability $\rho^{*}$ is the minimal solution in $(0,1]$ to the equation $1-\rho=f_{F}(1-\rho)$.

Here $f_{F}(x)=\mathbf{E}\left[x^{Z}\right], Z$ distribited as $F(x)$. Hence, we will prove that, for the process $\mathscr{B} P$ with offspring distribution

$\mathbf{C B}(n, m, p)$,

there is a $\rho^{*} \epsilon(2 \epsilon / 1+\gamma+(3+\gamma) \epsilon, 2 \epsilon / 1+\gamma-$

$(10 \gamma+10) \epsilon)$ such that $g\left(\rho^{*}\right)=0$, where

$$
\begin{aligned}
g(\rho) & =\rho-1+f_{\mathbf{C B}}(1-\rho), f_{\mathbf{C B}} \\
& =\mathbf{E}\left[x^{X^{*}}\right], X^{*} \text { ditributed as } \mathbf{C B}(n, m, p) .
\end{aligned}
$$

In fact, $g(\rho)$ is continuous on $\left[2 \epsilon / \chi_{1}, 2 \epsilon / \chi_{2}\right]$, where

$$
\begin{gathered}
\chi_{1}:=1+\gamma+(3+\gamma) \epsilon, \\
\chi_{2}:=(1+\gamma)(1-10) \epsilon .
\end{gathered}
$$

By inequality (35), we have

$$
\begin{aligned}
g\left(\frac{2 \epsilon}{\chi_{1}}\right) & =\left(1-p+p\left(1-\frac{2 p \epsilon}{\chi_{1}}\right)^{n}\right)^{m}+\frac{2 \epsilon}{\chi_{1}}-1 \\
& \leq{ }^{(35)}\left(1-p+p\left(1-\frac{2 n p \epsilon}{\chi_{1}}+\frac{2 n^{2} p^{2} \epsilon^{2}}{\chi_{1}^{2}}\right)\right)^{m}+\frac{2 \epsilon}{\chi_{1}}-1 \\
& =\left(1-\frac{2 n p^{2} \epsilon}{\chi_{1}}+\frac{2 n^{2} p^{3} \epsilon^{2}}{\chi_{1}^{2}}\right)^{m}+\frac{2 \epsilon}{\chi_{1}}-1 \\
& \leq{ }^{(35)} 1-\frac{2 m n p^{2} \epsilon}{\chi_{1}}+\frac{2 m n^{2} p^{3} \epsilon^{2}}{\chi_{1}^{2}}+\frac{2 m^{2} n^{2} p^{4} \epsilon^{2}}{\chi_{1}^{2}}\left(1-\frac{n p \epsilon}{\chi_{1}}\right)^{2}+\frac{2 \epsilon}{\chi_{1}}-1 \\
& \leq \frac{2 \epsilon}{\chi_{1}}-\frac{2 m n p^{2} \epsilon}{\chi_{1}}+\frac{2 m n^{2} p^{3} \epsilon^{2}}{\chi_{1}^{2}}+\frac{2 n^{2} m^{2} p^{4} \epsilon^{2}}{\chi_{1}^{2}}
\end{aligned}
$$




$$
\begin{aligned}
& =-\frac{2 \epsilon^{3}(1-\epsilon)}{\chi_{1}^{2}}<0 \\
& g\left(\frac{2 \epsilon}{\chi_{2}}\right)=\left(1-p+p\left(1-\frac{2 p \epsilon}{\chi_{2}}\right)^{n}\right)^{m}+\frac{2 \epsilon}{\chi_{2}}-1 \\
& { }^{(35)}\left(1-p+p\left(1-\frac{2 n p \epsilon}{\chi_{2}}+\frac{2 n^{2} p^{2} \epsilon^{2}}{\chi_{2}^{2}}-\frac{8 n^{3} p^{3} \epsilon^{3}}{\chi_{2}^{3}}\right)\right)^{m}+\frac{2 \epsilon}{\chi_{2}}-1 \\
& =\left(1-\frac{2 n p^{2} \epsilon}{\chi_{2}}+\frac{2 n^{2} p^{3} \epsilon^{2}}{\chi_{2}^{2}}-\frac{8 n^{3} p^{4} \epsilon^{3}}{\chi_{2}^{3}}\right)^{m}+\frac{2 \epsilon}{\chi_{2}}-1 \\
& { }^{(35)} 1-\frac{2 m n p^{2} \epsilon}{\chi_{2}}+\frac{2 m n^{2} p^{3} \epsilon^{2}}{\chi_{2}^{2}}-\frac{8 m n^{3} p^{4} \epsilon^{3}}{\chi_{2}^{3}}+\frac{2 \epsilon}{\chi_{2}}-1 \\
& +\frac{m^{2}}{2}\left(\frac{2 n p^{2} \epsilon}{\chi_{2}}-\frac{2 n^{2} p^{3} \epsilon^{2}}{\chi_{2}^{2}}+\frac{8 n^{3} p^{4} \epsilon^{3}}{\chi_{2}^{3}}\right)^{2}-m^{3}\left(\frac{2 n p^{2} \epsilon}{\chi_{2}}-\frac{2 n^{2} p^{3} \epsilon^{2}}{\chi_{2}^{2}}+\frac{8 n^{3} p^{4} \epsilon^{3}}{\chi_{2}^{3}}\right)^{3} \\
& =-\frac{2 \epsilon^{2}}{\chi_{2}}+\frac{2 \epsilon^{2}(1+\epsilon) \gamma}{\chi_{2}^{2}}+\frac{2 \epsilon^{2}(1+\epsilon)^{2}}{\chi_{2}^{2}}\left(1-\frac{\gamma \epsilon}{\chi_{2}}+\frac{4 \gamma^{2} \epsilon^{2}}{\chi_{2}^{2}}\right)^{2} \\
& -\frac{8 \epsilon^{3}(1+\epsilon)^{3}}{\chi_{2}^{3}}\left(1-\frac{\gamma \epsilon}{\chi_{2}}+\frac{4 \gamma^{2} \epsilon^{2}}{\chi_{2}^{2}}\right)^{3}-\frac{8 \epsilon^{3}(1+\epsilon) \gamma^{2}}{\chi_{2}^{3}} \\
& =-\frac{2 \epsilon^{2}(1-(11 \gamma+10) \epsilon)}{\chi_{2}^{2}}+\frac{2 \epsilon^{2}(1+\epsilon)^{2}}{\chi_{2}^{2}}\left(1-\frac{\gamma \epsilon}{\chi_{2}}+\frac{4 \gamma^{2} \epsilon^{2}}{\chi_{2}^{2}}\right)^{2} \\
& -\frac{8 \epsilon^{3}(1+\epsilon)}{\chi_{2}^{3}}\left[\gamma^{2}+(1+\epsilon)^{2}\left(1-\frac{\gamma \epsilon}{\chi_{2}}+\frac{4 \gamma^{2} \epsilon^{2}}{\chi_{2}^{2}}\right)^{3}\right] \\
& \geq-\frac{2 \epsilon^{2}(1-(11 \gamma+10) \epsilon)}{\chi_{2}^{2}}+\frac{2 \epsilon^{2}(1+\epsilon)^{2}}{\chi_{2}^{2}}\left(1-\frac{\gamma \epsilon}{\chi_{2}}\right)^{2}-\frac{8 \epsilon^{3}(1+\epsilon)}{\chi_{2}^{3}}\left[\gamma^{2}+(1+\epsilon)^{2}\right] \\
& \geq-\frac{2 \epsilon^{2}(1-(11 \gamma+10) \epsilon)}{\chi_{2}^{2}}+\frac{2 \epsilon^{2}}{\chi_{2}^{2}}\left(1-\frac{\gamma \epsilon}{\chi_{2}}\right)^{2}-\frac{8 \epsilon^{3}(1+\epsilon)}{\chi_{2}^{3}}\left[\gamma^{2}+(1+\epsilon)^{2}\right] \\
& \geq \frac{2 \epsilon^{3}}{\chi_{2}^{2}}\left[(11 \gamma+10)-\frac{2 \gamma}{\chi_{2}}\right]-\frac{8 \epsilon^{3}(1+\epsilon)}{\chi_{2}^{3}}\left[\gamma^{2}+(1+\epsilon)^{2}\right] \\
& =\frac{2 \epsilon^{3}}{\chi_{2}^{3}}\left[(11 \gamma+10) \chi_{2}-2 \gamma-4\left(\gamma^{2}+(1+\epsilon)^{2}\right)(1+\epsilon)\right]>0 \text {. }
\end{aligned}
$$

Hence, the claim holds by the Intermediate Value Theorem.
(2) Let $D^{*}$ be the number of children for each vertex in the dual processes $\mathscr{B} P^{*}$.

$$
\begin{aligned}
\mathbf{E}\left[D^{*}\right] & =\sum_{k \geq 0} k q_{k}=\sum_{k \geq 0} k p_{k}\left(\eta^{*}\right)^{k-1}=f_{\mathbf{C B}}{ }^{\prime}\left(\eta^{*}\right) \\
& =n m p^{2}\left(1-p+p\left(1-p \rho^{*}\right)^{n}\right)^{m-1}\left(1-p \rho^{*}\right)^{n-1} \\
& \leq{ }^{(35)} n m p^{2}\left(1-p+p\left(1-n p \rho^{*}+\frac{n^{2} p^{2}\left(\rho^{*}\right)^{2}}{2}\right)\right)^{m-1}\left(1-(n-1) p \rho^{*}+\frac{(n-1)^{2} p^{2}\left(\rho^{*}\right)^{2}}{2}\right)
\end{aligned}
$$




$$
\begin{aligned}
\leq & { }^{(35)} n m p^{2}\left(1-(m-1) n p^{2} \rho^{*}+\frac{(m-1) n^{2} p^{3}\left(\rho^{*}\right)^{2}}{2}+\frac{(m-1)^{2} n^{2} p^{4}\left(\rho^{*}\right)^{2}}{2}\right) \\
& \times\left(1-(n-1) p \rho^{*}+\frac{(n-1)^{2} p^{2}\left(\rho^{*}\right)^{2}}{2}\right) \\
\leq & (1+\epsilon)\left(1-\frac{2 \epsilon}{1+\gamma}+O\left(\epsilon^{2}\right)\right)\left(1-\frac{2 \gamma \epsilon}{1+\gamma}+O\left(\epsilon^{2}\right)\right) \\
= & 1-\epsilon+O\left(\epsilon^{2}\right) .
\end{aligned}
$$

Therefore,

$$
\begin{gathered}
\mathbf{E}\left(\left|B P_{F}^{*}\right|\right)=1+\mathbf{E}\left(D^{*}\right)+\left[\mathbf{E}\left(D^{*}\right)\right]^{2}+\left[\mathbf{E}\left(D^{*}\right)\right]^{3} \\
+\cdots \stackrel{(43)}{=} \frac{1}{1-\mathbf{E}\left(D^{*}\right)} \leq \frac{2}{\epsilon}
\end{gathered}
$$

(3) Denote the event that $\mathscr{B} P$ survives by $\mathcal{S}$ and the event that it dies out by $\mathcal{S}^{c}$

$$
\begin{aligned}
\mathbf{P}(|\mathscr{B} P| \geq L) & =\mathbf{P}(\mathcal{S}) \mathbf{P}(|\mathscr{B} P| \geq L \mid \mathcal{S})+\mathbf{P}\left(\mathcal{S}^{c}\right) \mathbf{P}\left(|\mathscr{B} P| \geq L \mid \mathcal{S}^{c}\right) \\
& =\mathbf{P}(\mathcal{S})+\mathbf{P}\left(\mathcal{S}^{c}\right) \mathbf{P}\left(\left|\mathscr{B} P^{*}\right| \geq L\right) \\
& \leq \frac{(2+o(1)) \epsilon}{1+\gamma}+\frac{\mathbf{E}\left(\left|\mathscr{B} P^{*}\right|\right)}{L} \leq(1+o(1))\left(\frac{2 \epsilon}{1+\gamma}+\frac{2}{\epsilon L}\right) .
\end{aligned}
$$

Let $S_{L}$ be the set of all vertices in the components that contain at least $L$ vertices, and let $s_{L}$ be the cardinality of this set. We have the following.

Lemma 8. Let $L=L(n)$ be a real function satisfying $\epsilon^{2} L \longrightarrow \infty$.

$$
\mathbf{E}\left(s_{L}\right) \leq \frac{(2+o(1)) \in n}{1+\gamma} .
$$

Proof. By Lemma 7,

$$
\begin{aligned}
\mathbf{E}\left(s_{L}\right) & =\sum_{v \in V} \mathbf{P}\left(\left|C_{v}\right| \geq L\right)=n \mathbf{P}\left(\left|C_{v}\right| \geq L\right) \leq n \mathbf{P}(|\mathscr{B} P| \geq L) \\
& \leq n(1+o(1))\left(\frac{2 \epsilon}{1+\gamma}+\frac{2}{\epsilon L}\right)=\frac{(2+o(1)) \epsilon n}{1+\gamma} .
\end{aligned}
$$

In the next lemma, we prove the size of large components is concentrated around its expectation.

Lemma 9. Suppose that $L=L(n)$ satisfies $\epsilon^{2} L \longrightarrow \infty$ and $L=o(\epsilon n)$. Then w.h.p. $s_{L}=(2+o(1)) \epsilon /(1+\gamma)$.

The proof of Lemma 9 follows the part of the proof of Lemma 7 in [14] verbatim except that some constants need to be changed. We omit it here.
Proof of Upper Bound in Weakly Supercritical Case in Theorem 1. Let $\omega(n)=\epsilon n^{1 / 3}$ and $L=\epsilon n / \log \omega(n)$. Then the upper bound of (1) in Theorem 1 is obtained from Lemma 9.

3.2. Upper Bound in the Weakly Subcritical Regime. Outline of the proof is as follows: We first use exploration process $\left\{Y_{t}^{*}\right\}_{t \geq 0}$ to explore the components of $\mathscr{B}(n, m, p)$. Then, for the upper bound of $\left|\mathscr{C}_{1}\right|$, we only need to give the upper bound on the stop time $T^{*}$ as $\left|\mathscr{C}\left(v_{0}\right)\right|=T^{*}$. For this, we follow the ideas in [[32], Proposition 1]. Recall Lemma 10 .

Lemma 10 (see Lemma 8 in [32]). Let $\eta$ be an integer-valued random variable with $\mathbf{E}\left(\eta^{2}\right)<\infty$ such that, for any integer $h \geq 2, \mathbf{P}(\eta \in h \mathbb{Z})<1$. Let $\left\{\eta_{i}\right\}_{i=1}^{\infty}$ be a sequence of the i.i.d. random variables distributed as $\eta$ and

$$
W_{t}=W_{0}+\sum_{i=1}^{t} \eta_{i}, \quad t \in\{0,1,2, \ldots,\}
$$

where $W_{0}$ is an integer constant. Define $\tau:=\min \left\{t: W_{t}=0\right\}$. If $\theta_{0}>0$ and it satisfies $\mathbf{E}\left(\eta e^{\theta_{0} \eta}\right)=0$, then, for any integer $\ell \geq 1$,

$$
\mathbf{P}(\tau=\ell)=\Theta\left(\ell^{-3 / 2} \phi\left(\theta_{0}\right)^{\ell}\right)
$$

where $\phi(\theta)=\mathbf{E}\left(e^{\theta \eta}\right)$ and the constants in $\Theta$ depend only on $\eta$ and $W_{0}$ but not $\ell$. 
Proof of Upper Bound in Weakly Subcritical Case in Theorem 1. To utilize Lemma 10, set

$$
S_{t}=1+\sum_{i=1}^{t}\left(\xi_{i}-1\right),
$$

$$
\begin{aligned}
\mathbf{E}[\varsigma] & =\mathbf{E}\left(\xi_{i}-1\right)=f_{X^{*}}^{\prime}(1)-1=-\epsilon, \\
\mathbf{E}\left[\varsigma^{2}\right] & =f_{X^{*}}^{(2)}(1)-f_{X^{*}}^{\prime}(1)=(1-\epsilon)(1+\gamma)+\epsilon^{2}(1+o(1)), \\
\mathbf{E}\left[\varsigma^{3}\right] & =f_{X^{*}}^{(3)}(1)+f_{X^{*}}^{\prime}(1)-1=\gamma^{2}+3 \gamma+1-\left(\gamma^{2}+3 \gamma+3\right) \epsilon+\epsilon^{2}(1+o(1)) .
\end{aligned}
$$

Set $\quad a(n)=\epsilon /(1+\gamma)(1-\epsilon)+\left(\gamma^{2}+3 \gamma+2\right) \epsilon \quad$ and $b(n)=\epsilon /(1+\gamma)(1-\epsilon)-2 \epsilon$. Define $\quad F(\theta)=\mathbf{E}\left[\varsigma e^{\theta \varsigma}\right]$ for $\theta \in[a(n), b(n)]$. In the following, we check that there is $\theta_{0}$ such that

$$
\begin{array}{r}
a(n)<\theta_{0}<b(n), \\
F\left(\theta_{0}\right)=\mathbf{E}\left(\varsigma e^{\theta_{0} \varsigma}\right)=0 .
\end{array}
$$

$$
\begin{aligned}
\mathbf{E}\left[\varsigma e^{b(n) \varsigma}\right] & =-e^{-b(n)} p_{-1}+e^{b(n)} p_{1}+2 e^{2 b(n)} p_{2}+\cdots+(n-1) e^{(n-1) b(n)} p_{n-1} \\
& \stackrel{(53)}{=} e^{-b(n)}\left\{-\epsilon-\sum_{i=1}^{n-1} i p_{i}\right\}+\sum_{i=1}^{n-1} i e^{i b(n)} p_{i} \\
& =-\epsilon e^{-b(n)}+\sum_{i=1}^{n-1} i p_{i}\left(e^{i b(n)}-e^{-b(n)}\right) \\
& \geq-\epsilon e^{-b(n)}+b(n) \sum_{i=1}^{n-1} i(i+1) p_{i} \\
& \geq-\epsilon\left(1-b(n)+b^{2}(n)\right)+b(n)\left[\mathbf{E}\left(\varsigma^{2}\right)+\mathbf{E}(\varsigma)\right] \\
& =-\epsilon+b(n)[(1+\gamma)(1-\epsilon)-\epsilon+o(\epsilon)]>0 .
\end{aligned}
$$

by (53)-(55), we can obtain

$$
\begin{aligned}
\mathbf{E}\left[\varsigma e^{a(n) \varsigma}\right] & =\sum_{i=-1}^{n-1}\left(e^{a(n)}\right)^{i} i p_{i} \leq{ }^{(36)} \sum_{i=-1}^{n-1}\left(1+a(n)+a^{2}(n)\right)^{i} i p_{i} \\
& \leq \sum_{i=-1}^{(37)}\left(1+i a(n)+i a^{2}(n)+\frac{2 i^{2} a^{2}(n)}{3}(1+a(n))^{2}\right) i p_{i} \\
& =\sum_{i=-1}^{n-1} k p_{k}+a(n) \sum_{i=-1}^{n-1} i^{2} p_{i}+\frac{2 a^{2}(n)(1+a(n))^{2}}{3} \sum_{i=-1}^{n-1} i^{3} p_{i}
\end{aligned}
$$




$$
\begin{aligned}
& \leq \mathbf{E}(\varsigma)+a(n) \mathbf{E}\left(\varsigma^{2}\right)+a^{2}(n) \mathbf{E}\left(\varsigma^{3}\right) \\
& \leq-\epsilon+\frac{\epsilon[(1+\gamma)(1-\epsilon)+o(\epsilon)]}{(1+\gamma)(1-\epsilon)+\left(\gamma^{2}+3 \gamma+2\right) \epsilon}+\frac{\epsilon^{2}\left(\gamma^{2}+3 \gamma+1\right)}{\left[(1+\gamma)(1-\epsilon)+\left(\gamma^{2}+3 \gamma+2\right) \epsilon\right]^{2}} \\
& \leq-\frac{\left(\gamma^{2}+3 \gamma+2\right) \epsilon^{2}+o\left(\epsilon^{2}\right)}{(1+\gamma)(1-\epsilon)+\left(\gamma^{2}+3 \gamma+2\right) \epsilon}+\frac{\epsilon^{2}\left(\gamma^{2}+3 \gamma+1\right)}{\left[(1+\gamma)(1-\epsilon)+\left(\gamma^{2}+3 \gamma+2\right) \epsilon\right]^{2}}<0 .
\end{aligned}
$$

Third, it is obvious that $F(\theta)$ is continuous on $[a(n), b(n)]$. Hence, together with (57) and (58), this implies (56) holds by the Intermediate Value Theorem.

Now we estimate $\mathbf{E}\left[e^{\theta_{0} \varsigma}\right]$. By (53) and (54), we can deduce that

$$
\begin{aligned}
\mathbf{E}\left[e^{\theta_{0} \varsigma}\right] & =\sum_{k=-1}^{n-1}\left(e^{\theta_{0}}\right)^{k} p_{k} \leq{ }^{(36)} \sum_{k=-1}^{n-1}\left(1+\theta_{0}+\theta_{0}^{2}\right)^{k} p_{k} \\
& \leq{ }^{(37)} \sum_{k=-1}^{n-1}\left(1+k\left(\theta_{0}+\theta_{0}^{2}\right)+\frac{2 k^{2}}{3}\left(\theta_{0}+\theta_{0}^{2}\right)^{2}\right) p_{k} \\
& =\sum_{k=-1}^{n-1} p_{k}+\left(\theta_{0}+\theta_{0}^{2}\right) \sum_{k=-1}^{n-1} k p_{k}+\frac{2\left(\theta_{0}+\theta_{0}^{2}\right)^{2}}{3} \sum_{k=-1}^{n-1} k^{2} p_{k} \\
& =1+\left(\theta_{0}+\theta_{0}^{2}\right) \mathbf{E}(\varsigma)+\frac{2\left(\theta_{0}+\theta_{0}^{2}\right)^{2}}{3} \mathbf{E}\left(\varsigma^{2}\right) \\
& =1-\frac{\epsilon^{2}(n)}{3(1+\gamma)}+o\left(\epsilon^{2}\right) .
\end{aligned}
$$

Suppose that there are $\ell$ active vertices and $k$ explored vertices have been explored at the time step $t$ in the exploration process on $\mathscr{B}(n, m, p)$. We can see that the number of newly explored vertices $X_{t}^{*}$ through the current vertex, say $v$, has the distribution $\mathbf{C B}(n-k-\ell, m, p)$. Therefore, by Lemma $6, X_{t}^{*}<\xi_{t}$, where $\xi_{t}$ is distributed as $\mathbf{C B}(n, m, p)$. That is to say, for all $k \leq \min \left\{T^{*}, n\right\}$,

$$
\sum_{i=1}^{k} X_{i}^{*} \prec \sum_{i=1}^{k} \xi_{i}
$$

where $T^{*}=\min \left\{t: Y_{t}^{*}=0\right\}$. Let $\mathscr{C}_{t}\left(v_{0}\right)$ denote total explored vertices which are explored from time 0 to $t$ by the exploration process on $\mathscr{B}(n, m, p)$ starting from vertex $v_{0}$. Define $\tau_{S}=\min \left\{t: S_{t}=0, t \leq n\right\}$. Note that

$$
\left|\mathscr{C}_{t}\left(v_{0}\right)\right|=1+\sum_{i=1}^{t}\left(X_{i}^{*}-1\right) \text { and } S_{t}=1+\sum_{i=1}^{t}\left(\xi_{i}-1\right) \text {. }
$$

This means $\left|\mathscr{C}_{t}\left(v_{0}\right)\right|<S_{t}$ implying $T^{*} \prec \tau_{s}$. Therefore, by Lemma 10 ,

$$
\begin{aligned}
\mathbf{P}\left(\left|\mathscr{C}\left(v_{0}\right)\right| \geq M_{0}\right) & =\mathbf{P}\left(T^{*} \geq M_{0}\right) \leq \mathbf{P}\left(\tau_{S} \geq M_{0}\right) \\
& =\sum_{\ell \geq M_{0}} \mathbf{P}\left(\tau_{S}=\ell\right) \\
& =\sum_{\ell \geq M_{0}} O\left(\ell^{-3 / 2}\left(1-\frac{\epsilon^{2}}{3(1+\gamma)}+o\left(\epsilon^{2}\right)\right)^{\ell}\right) .
\end{aligned}
$$

Set $M_{0}=4(1+\gamma) \epsilon^{-2}(n) \log \left(n \epsilon^{3}(n)\right)$. By the inequality $1-x \leq e^{-x}$, for $x \geq 0$,

$$
\begin{aligned}
\mathbf{P}\left(\tau_{S} \geq M_{0}\right) & =O\left(M_{0}^{(-3) / 2}\right) \sum_{\ell \geq M_{0}}\left(1-\frac{\epsilon^{2}}{3(1+\gamma)}+o\left(\epsilon^{2}\right)\right)^{\ell} \\
& =O\left(\epsilon^{-2} M_{0}^{(-3) / 2}\right)\left(1-\frac{\epsilon^{2}}{3(1+\gamma)}+o\left(\epsilon^{2}\right)\right)^{M_{0}} \\
& =O\left(\epsilon^{-2} M_{0}^{(-3) / 2}\right) \exp \left\{-\frac{M_{0} \epsilon^{2}}{3(1+\gamma)}+o\left(M_{0} \epsilon^{2}\right)\right\} \\
& =O\left(\epsilon\left(\log n \epsilon^{3}\right)^{(-3) / 2}\left(n \epsilon^{3}\right)^{-(4 / 3)+o(1)}\right) .
\end{aligned}
$$

Denote $Z_{\geq k}=\sum_{v \in V} 1_{\{|\mathscr{C}(v)| \geq k\}}$.

$$
\begin{aligned}
\mathbf{P}\left(\left|\mathscr{C}_{1}\right| \geq M_{0}\right) & =\mathbf{P}\left(Z_{\geq M_{0}} \geq M_{0}\right) \leq \frac{\mathbf{E}\left(Z_{\geq M_{0}}\right)}{M_{0}}=\frac{\sum_{v \in V} \mathbf{E}\left(1_{\left\{|\mathscr{C}(v)| \geq M_{0}\right\}}\right)}{M_{0}}=\frac{n \mathbf{P}\left(\left|\mathscr{C}\left(v_{0}\right)\right| \geq M_{0}\right)}{M_{0}} \\
& \leq \frac{n \mathbf{P}\left(\tau_{S} \geq M_{0}\right)}{M_{0}}=O\left(\left(n \epsilon^{3}\right)^{-(1 / 3-o(1))}\left(\log n \epsilon^{3}\right)^{-5 / 2}\right)=o(1) .
\end{aligned}
$$




\section{Data Availability}

Data sharing is not applicable to this article as no datasets were generated or analyzed during the current study.

\section{Conflicts of Interest}

The authors declare that there are no conflicts of interest regarding the publication of this study.

\section{Acknowledgments}

The second author was supported partially by the NSFC (no. 11961015), the GXNSF (no. 2018GXNSFAA050031), and the startup grant from Guilin University of Technology.

\section{References}

[1] K. B. Singer-Cohen, "Random intersection graphs," Ph.D. Thesis, John Hopkins Univresity, Baltimore, MA, USA, 1999.

[2] M. Karoński, E. R. Scheinerman, and K. B. Singer-Cohen, "On random intersection graphs: the subgraph problem," Combinatorics, Probability and Computing, vol. 8, pp. 131-159, 1999.

[3] E. Godehardt and J. Jaworski, "Two models of random intersection graphs for classification," in Exploratory Data Analysis in Empirical Research, Studies in Classification, Data Analysis, and Knowledge Organization, M. Schwaiger and O. Opitz, Eds., Springer, Berlin, Germany, pp. 67-81, 2003.

[4] E. Godehardt, J. Jaworski, and K. Rybarczyk, "Random intersection graphs and classification," in Advances in Data Analysis, Studies in Classification, Data Analysis, and Knowledge Organization, R. Decker and H. J. Lenz, Eds., Springer, Berlin, Germany, pp. 67-74, 2007.

[5] M. Bloznelis, "Degree and clustering coefficient in sparse random intersection graphs," Annals of Applied Probability, vol. 23, no. 3, pp. 1254-1289, 2013.

[6] M. Deijfen and W. Kets, "Random intersection graphs with tunable degree distribution and clustering," Probability in the Engineering and Informational Sciences, vol. 23, no. 4, pp. 661-674, 2009.

[7] J. Zhao, O. Yağan, and V. Gligor, "Secure $k$-connectivity in wireless sensor networks under an on/off channel model," in Proceedings of the 2013 IEEE International Symposium on Information Theory, pp. 2790-2794, Istanbul, Turkey, July 2013.

[8] F. G. Ball, D. J. Sirl, and P. Trapman, "Epidemics on random intersection graphs," Annals of Applied Probability, vol. 24, pp. 1081-1128, 2014.

[9] J. Zhao, O. Yağan, and V. Gligor, "Random intersection graphs and their applications in security, wireless communication, and social networks," in Proceedings of the Information Theory and Applications Workshop (ITA 2015), San Diego, CA, USA, February 2015.

[10] M. Behrisch, "Component evolution in random intersection graphs," Electronic Journal of Combinatorics, vol. 14, no. 1, p. P17, 2007.

[11] A. N. Lagerås and M. Lindholm, "A note on the component structure in random intersection graphs with tunable clustering," Electronic Journal of Combinatorics, vol. 15, p. N10, 2007.

[12] M. Bloznelis and L. Leskelä, "Clustering and percolation on superpositions of Bernoulli random graphs," 2019, https:// arxiv.org/abs/1912.13404.
[13] B. Wang, L. Wang, and K. Xiang, "The largest component in critical random intersection graphs," Discussiones Mathematicae Graph Theory, vol. 38, no. 4, pp. 921-946, 2018.

[14] B. Wang and L. Zou, "Size of the largest component in a critical graph," Mathematical Problems in Engineering, vol. 2019, Article ID 1731802, 2019.

[15] L. Federico, "Critical scaling limits of the random intersection graph,” 2019, https://arxiv.org/abs/1910.13227v1.

[16] K. Rybarczyk, "Sharp threshold functions for random intersection graphs via a coupling method," Electronic Journal of Combinatorics, vol. 18, p. P36, 2011.

[17] T. Johannson, "The giant component of the random bipartite graph," Master's Thesis, Chalmers University of Technology, Gothenburg, Sweden, 2012.

[18] U. D. Ambroggio, "An elementary approach to component sizes in some cirtical random graphs," http://arxiv.org/abs/ $2101.06625 \mathrm{v} 1$.

[19] N. Alon and J. H. Spencer, The Probabilistic Method, John Wiley \& Sons, Hoboken, NJ, USA, 3nd edition, 2008.

[20] I. Ben-Eliezer, M. Krivelevich, and B. Sudakov, "Long cycles in subgraphs of (pseudo) random directed graphs," Journal of Graph Theory, vol. 70, no. 3, pp. 284-296, 2012.

[21] M. Krivelevich and B. Sudakov, "The phase transition in random graphs: a simple proof," Random Structures and Algorithms, vol. 43, no. 2, pp. 131-138, 2013.

[22] A. Joseph, "The component sizes of a critical random graph with given degeee sequence," Annals of Applied Probability, vol. 24, pp. 2560-2594, 2014.

[23] O. Cooley, M. Kang, and Y. Person, "Largest components in random hypergraphs," Combinatorics, Probability and Computing, vol. 27, no. 5, pp. 741-762, 2018.

[24] T. Bohman, "The triangle-free process," Advances in Mathematics, vol. 221, no. 5, pp. 1653-1677, 2009.

[25] A. Nachmias and Y. Peres, "Component sizes of the random graph outside the scaling window," ALEA. Latin American Journal of Probability and Mathematical Statistics, vol. 3, pp. 133-142, 2007.

[26] R. Durrett, Probability Theory and Examples, Cambridge University Press, Cambridge, UK, 4th edition, 2010.

[27] B. Bollobás, S. Janson, and O. Riordan, "The phase transition in inhomogeneous random graphs," Random Structures and Algorithms, vol. 31, no. 1, pp. 3-122, 2007.

[28] R. v. d. Hofstad, Random Graphs and Complex Networks (Volume 1), Cambridge University Press, Cambridge, UK, 2017.

[29] B. Bollobás and O. Riordan, "A simple branching process approach to the phase transition in $G_{n, p}$," Electronic Journal of Combinatorics, vol. 19, p. P21, 2012.

[30] M. Kang, C. Koch, and A. Pachón, "The phase transition in multitype binomial random graphs," SIAM Journal on Discrete Mathematics, vol. 29, no. 2, pp. 1042-1064, 2015.

[31] T. E. Harris, The Theory of Branching Process, Springer-Verlag, Berlin, Germany, 1963.

[32] A. Nachmias and Y. Peres, "Critical percolation on random regular graphs," Random Structures and Algorithms, vol. 36, no. 2, pp. 111-148, 2010. 